\title{
Peripheral blood eosinophils: a surrogate marker for airway eosinophilia in stable COPD
}

\author{
This article was published in the following Dove Press journal: \\ International Journal of COPD \\ I July 2016 \\ Number of times this article has been viewed
}

\author{
Netsanet A Negewo ${ }^{1,2}$ \\ Vanessa M McDonald ${ }^{1-4}$ \\ Katherine J Baines ${ }^{1,2}$ \\ Peter AB Wark ${ }^{1-3}$ \\ Jodie L Simpson ${ }^{1,2}$ \\ Paul W Jones ${ }^{5}$ \\ Peter G Gibson ${ }^{1-3}$
}

'Priority Research Centre for Healthy Lungs, Faculty of Health and Medicine, The University of Newcastle, Callaghan, NSW, Australia; ${ }^{2}$ Hunter Medical Research Institute, Faculty of Health and Medicine, The University of Newcastle, Callaghan, NSW, Australia; ${ }^{3}$ Department of Respiratory and Sleep Medicine, John Hunter Hospital, Newcastle, NSW, Australia; ${ }^{4}$ School of Nursing and Midwifery, Faculty of Health and Medicine, The University of Newcastle, Callaghan, NSW, Australia; ${ }^{5}$ nstitute for Infection and Immunity, St George's, University of London, London, UK

Correspondence: Netsanet A Negewo Hunter Medical Research Institute, Level 2 West Wing, Locked Bag 1000, New Lambton, NSW 2305, Australia

Tel +6I 240420762

Fax +6I 240420046

Email netsanet.negewo@uon.edu.au
Introduction: Sputum eosinophilia occurs in approximately one-third of stable chronic obstructive pulmonary disease (COPD) patients and can predict exacerbation risk and response to corticosteroid treatments. Sputum induction, however, requires expertise, may not always be successful, and does not provide point-of-care results. Easily applicable diagnostic markers that can predict sputum eosinophilia in stable COPD patients have the potential to progress COPD management. This study investigated the correlation and predictive relationship between peripheral blood and sputum eosinophils. It also examined the repeatability of blood eosinophil counts.

Methods: Stable COPD patients $(n=141)$ were classified as eosinophilic or noneosinophilic based on their sputum cell counts ( $\geq 3 \%$ ), and a cross-sectional analysis was conducted comparing their demographics, clinical characteristics, and blood cell counts. Receiver operating characteristic curve analysis was used to assess the predictive ability of blood eosinophils for sputum eosinophilia. Intraclass correlation coefficient was used to examine the repeatability of blood eosinophil counts.

Results: Blood eosinophil counts were significantly higher in patients with sputum eosinophilia ( $\mathrm{n}=45$ ) compared to those without $\left(0.3 \times 10^{9} / \mathrm{L}\right.$ vs $\left.0.15 \times 10^{9} / \mathrm{L} ; P<0.0001\right)$. Blood eosinophils correlated with both the percentage $(\rho=0.535 ; P<0.0001)$ and number of sputum eosinophils $(\rho=0.473 ; P<0.0001)$. Absolute blood eosinophil count was predictive of sputum eosinophilia (area under the curve $=0.76,95 \%$ confidence interval $[\mathrm{CI}]=0.67-0.84 ; P<0.0001$ ). At a threshold of $\geq 0.3 \times 10^{9} / \mathrm{L}$ (specificity $=76 \%$, sensitivity $=60 \%$, and positive likelihood ratio $=2.5$ ), peripheral blood eosinophil counts enabled identification of the presence or absence of sputum eosinophilia in $71 \%$ of the cases. A threshold of $\geq 0.4 \times 10^{9} / \mathrm{L}$ had similar classifying ability but better specificity $(91.7 \%)$ and higher positive likelihood ratio (3.7). In contrast, $\geq 0.2 \times 10^{9} / \mathrm{L}$ offered a better sensitivity (91.1\%) for ruling out sputum eosinophilia. There was a good agreement between two measurements of blood eosinophil count over a median of 28 days (intraclass correlation coefficient $=0.8 ; 95 \% \mathrm{CI}=0.66-0.88 ; P<0.0001)$.

Conclusion: Peripheral blood eosinophil counts can help identify the presence or absence of sputum eosinophilia in stable COPD patients with a reasonable degree of accuracy.

Keywords: sputum eosinophilia, diagnostic accuracy, chronic obstructive pulmonary disease, stability of eosinophil counts

\section{Introduction}

Airway eosinophilia, a hallmark feature of asthma, is now a recognized inflammatory pattern in chronic obstructive pulmonary disease (COPD). ${ }^{1-3}$ Eosinophilic COPD, defined as sputum eosinophils $\geq 3 \%$, is reported during acute exacerbations in up to $28 \%$ of cases, ${ }^{4}$ and interestingly, in periods of disease stability, it is seen in approximately $34 \%^{5}$ ( or $38 \%{ }^{6}$ ) of COPD patients. Airway eosinophilia is a reliable predictor 
of responsiveness to inhaled and oral corticosteroid therapies in COPD. ${ }^{6-9}$

The detection and measurement of airway eosinophilia mostly require the assessment of induced sputum. ${ }^{2}$ Although sputum induction is considered a direct and reliable method of assessing airway inflammation, it has a number of limitations..$^{10,11}$ In addition to being unsuitable for point-of-care testing, it requires expertise and may not be always successful (failure rate of up to $30 \%$ ). ${ }^{10,11}$ Due to these reasons, the search for minimally invasive and easily applicable diagnostic tools that can predict sputum eosinophilia in COPD and asthma has intensified..$^{410,12-15}$ The use of peripheral blood cell counts as a potential alternative is attracting profound interest owing to its ease of application in clinical practice. The ability of blood eosinophils to predict sputum eosinophilia in patients with asthma has been reported, with promising results. ${ }^{15-18}$ In COPD, however, very few studies have addressed this, particularly during clinical stability. A recent report in 20 COPD patients and 21 healthy controls has demonstrated the association between bronchial and blood eosinophil counts. ${ }^{19}$ Studies have also shown the potential ability of blood eosinophils to serve as a marker of response to corticosteroid treatments in exacerbating ${ }^{20}$ and stable ${ }^{21,22}$ COPD patients. The clinical characteristics of nonexacerbating COPD patients with persistently elevated levels of blood eosinophils ( $\geq 2 \%$ ) and their longitudinal changes during a follow-up period of 3 years have also been investigated. ${ }^{3}$ Nevertheless, studies examining the utility of blood eosinophils in detecting sputum eosinophilia in stable COPD are still lacking.

In this study, we hypothesized that peripheral blood eosinophils can serve as a promising surrogate marker for sputum eosinophilia in stable COPD. To test this hypothesis, a cross-sectional analytical study of 141 stable COPD patients was conducted with the aim of investigating the correlation and predictive relationship between peripheral blood and sputum eosinophils. In addition, the stability of peripheral blood eosinophil counts between two measurements over a median period of 28 days was examined.

\section{Methods}

\section{Study design}

A cross-sectional analytical study was conducted involving 141 patients with stable COPD (Figure 1). The data for 71 participants were obtained from our previously published studies. ${ }^{5,23,24}$ The remaining 70 participants were recruited from the respiratory ambulatory care clinics at John Hunter Hospital (Newcastle, Australia), the clinical research databases of the Priority Research Centre for Asthma and Respiratory Disease at the University of Newcastle and the

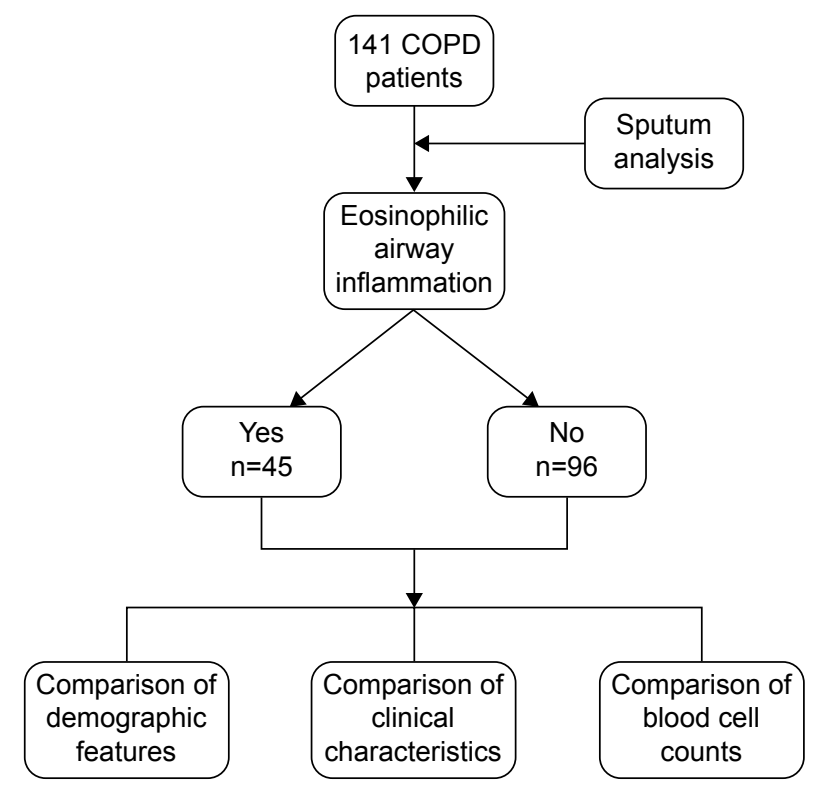

Figure I Study flowchart.

Hunter Medical Research Institute (Newcastle, Australia), and through community advertisement. All participants provided written informed consent, and ethics approval was obtained from the Human Ethics Research Committees of the Hunter New England Local Health District (12/12/12/3.06) and the University of Newcastle (H-2013-0010).

\section{Study participants}

Adults ( $\mathrm{n}=141)$ with stable COPD and paired blood and sputum cell counts, which were obtained from samples collected during the same visit, were included. COPD diagnosis was confirmed by incompletely reversible airflow limitation (postbronchodilator forced expiratory volume in 1 second $\left[\mathrm{FEV}_{1}\right]$ $<80 \%$ predicted and $\mathrm{FEV}_{1}$ to forced vital capacity [FVC] ratio of $<0.7$ ). Stable COPD was defined as no increase in bronchodilator use, no use of oral corticosteroids or antibiotics, no unscheduled doctor's visit, or no hospitalization due to COPD in the past 4 weeks. Participants were assessed for demographic features, lung function, airway and peripheral blood inflammatory cell counts, smoking history, body mass index, preceding year exacerbation history, medical history, dyspnea (modified Medical Research Council [mMRC]), ${ }^{25}$ comorbidities (Charlson Comorbidity Index), ${ }^{26}$ and health-related quality of life (St George Respiratory Questionnaire). ${ }^{27}$ The BODEx (body mass index, airflow obstruction, dyspnea, severe exacerbation) index was also calculated. ${ }^{28}$

\section{Spirometry}

Airflow limitation was assessed using spirometry (Medgraphics, CPFS/D ${ }^{\text {TM }}$ USB Spirometer, BreezeSuite v7.1, MGC 
Diagnostics, Saint Paul, MN, USA) to measure pre- and postbronchodilator $\mathrm{FEV}_{1}, \mathrm{FVC}$, and $\mathrm{FEV}_{1} / \mathrm{FVC}$ according to the standards of the American Thoracic Society. ${ }^{29}$ The third National Health and Nutrition Examination Survey reference equations were used to calculate percent predicted. ${ }^{30}$

\section{Sputum induction and analysis}

Sputum was induced using nebulized $4.5 \%$ saline in participants whose $\mathrm{FEV}_{1}$ was $\geq 1 \mathrm{~L}$, using our previously described methods. ${ }^{31}$ In those with $\mathrm{FEV}_{1}<1 \mathrm{~L}, 0.9 \%$ saline was used. Lower respiratory sputum portions were selected and dispersed using dithiothreitol, and total cell count viability was performed. Cytospins were prepared, stained (May-Grunwald-Giemsa), and a differential cell count was obtained from 400 nonsquamous cells. Sputum samples obtained from all participants had squamous cell contamination less than $80 \%$ and were deemed adequate for further analysis. ${ }^{32}$ We defined eosinophilic COPD as sputum eosinophil count of $\geq 3 \%$. $^{33}$

\section{Blood collection and analysis}

Peripheral venous blood was collected into Vacutainer ${ }^{\circledR}$ tubes (BD Worldwide, North Ryde, NSW, Australia). Full blood counts were performed using standardized methods on a Beckman Coulter LH series analyzer (Beckman Coulter Ltd, Brea, CA, USA), through Hunter Area Pathology Service (Newcastle, Australia). The stability of peripheral blood eosinophils between two measurements, approximately 28 days apart, was evaluated in 46 participants who had repeated measurements of blood cell counts.

\section{Statistical analysis}

Data were analyzed using Stata 13 (Stata Corporation, College Station, TX, USA). Results are reported as mean \pm standard deviation (SD) for normally distributed data and as median and interquartile range for nonparametric data. Student's $t$-test was used for comparisons of normally distributed data, and Wilcoxon rank sum test was used for skewed data. Comparison of categorical data was done using Fisher's exact test. Spearman's rank correlation coefficient was used to examine the association between absolute blood cell counts/ratios and sputum eosinophils. Receiver operating characteristic (ROC) curves were generated, and the area under the curve (AUC) was calculated to assess the predictive relationship between blood and sputum eosinophils. Intraclass correlation coefficient and Bland-Altman plot were used to determine the agreement between two measurements of blood eosinophil counts. All results were reported as significant when $P<0.05$.

\section{Results Clinical characteristics}

Table 1 presents the demographics and clinical characteristics of the 141 participants. Participants had a mean (SD) age of $69.8 \pm 7.7$ years and mean postbronchodilator predicted $\mathrm{FEV}_{1}$ of $57.5 \% \pm 17.9 \%$. There were $89(63.1 \%)$ males. Most of the participants $(116,82.3 \%)$ were ex-smokers with median pack-years of $37.5(13.8,62.5)$. In terms of GOLD (Global Initiative for Chronic Obstructive Lung Disease) grades, $11(7.8 \%)$ patients were in GOLD I, $74(52.5 \%)$ in GOLD II, $45(31.9 \%)$ in GOLD III, and $11(7.8 \%)$ in GOLD IV. With regard to the GOLD quadrants, using mMRC for symptom assessment, approximately half (53.9\%) of the participants were in Quadrant D and 24.8\% in Quadrant B. Over half $(52.5 \%)$ were "frequent exacerbators", having had two or more exacerbations in the past 12 months. Most (128, 90.8\%) of the participants were prescribed maintenance inhaled corticosteroids (ICS) or ICS and long-acting $\beta_{2}$ agonist (LABA) combination therapy (ICS/LABA), and of these, $102(72.3 \%$ of the total population) participants were also taking long-acting muscarinic antagonists (LAMA).

Eosinophilic airway inflammation (sputum eosinophil count $\geq 3 \%$ ) was present in $45(31.9 \%)$ participants. Clinical characteristics were similar between those with sputum eosinophilia and those without, except for higher BODEx score $(P=0.003)$ and more frequent high level of breathlessness $(\mathrm{mMRC} \geq 2)(P=0.01)$ in the latter.

\section{Peripheral blood cell counts/ratios}

Both the absolute number and percentage proportion of blood eosinophils were significantly higher in eosinophilic COPD compared with noneosinophilic COPD $\left(0.30 \times 10^{9} / \mathrm{L}\right.$ vs $0.15 \times 10^{9} / \mathrm{L}, P<0.0001$ and $3.95 \%$ vs $2.07 \%, P<0.0001$, respectively) (Table 2, Figure 2A). Noneosinophilic participants had significantly elevated blood neutrophil counts compared with the eosinophilic group $\left(5.3 \times 10^{9} / \mathrm{L}\right.$ vs $4.6 \times 10^{9} / \mathrm{L}$, $P=0.02)$. There was no difference in blood lymphocytes $(P=0.84)$ and total white blood cell counts $(P=0.32)$ between the two groups. Participants with sputum eosinophilia had significantly higher blood eosinophil/neutrophil ratio (ENR) and eosinophil/lymphocyte ratio (ELR), whereas those without had significantly higher neutrophil/lymphocyte ratio (Table 2, Figure 2B-D).

\section{Correlation between blood and sputum eosinophils}

A significant correlation was found between blood eosinophil counts and the proportion $(\rho=0.535 ; P<0.0001)$ (Figure 3A) and number of sputum eosinophils $(\rho=0.473 ; P<0.0001)$ 
Table I Demographics and clinical characteristics of the study population

\begin{tabular}{|c|c|c|c|c|}
\hline Variable & All participants $(n=|4|)$ & Eosinophilic ${ }^{a}(n=45)$ & Noneosinophilic ${ }^{\mathrm{b}}(\mathrm{n}=96)$ & $P$-value* \\
\hline Age (years) & $69.8 \pm 7.7$ & $68.5 \pm 8.0$ & $70.3 \pm 7.6$ & 0.20 \\
\hline Sex (male) & $89(63)$ & $30(67)$ & $59(61)$ & 0.58 \\
\hline Postbronchodilator FEV (\% predicted) & $57.5 \pm 17.9$ & $58.3 \pm 18.8$ & $57.1 \pm 17.6$ & 0.72 \\
\hline Postbronchodilator FEV (L) & $1.5(1.12,1.79)$ & $1.56(1.09,1.79)$ & $1.48(1.12,1.78)$ & 0.48 \\
\hline Postbronchodilator $\mathrm{FEV} / \mathrm{FVC}$ ratio (\%) & $56.55(43.31,65.57)$ & $55.26(44.4,62.65)$ & $57.12(42.56,65.92)$ & 0.46 \\
\hline Reversibility (L) & $0.04(0.006,0.10)$ & $0.04(0,0.08)$ & $0.05(0.008,0.11)$ & 0.48 \\
\hline Reversibility (\%) & $4.46(0.63,10.22)$ & $3.9(0.0,7.5)$ & $4.67(0.9,11.0)$ & 0.48 \\
\hline $\mathrm{CCl}$ score & $3.9 \pm 1.1$ & $3.8 \pm 1.0$ & $3.9 \pm 1.1$ & 0.71 \\
\hline Frequent vs nonfrequent exacerbators ${ }^{c}$ & 74 vs 67 (52.5 vs 47.5$)$ & 23 vs 22 (5I.I vs 48.9$)$ & 5 I vs 45 (53.I vs 46.9$)$ & 0.86 \\
\hline GOLD grade & & & & 1.00 \\
\hline 1 & II (7.8) & $3(6.7)$ & $8(8.3)$ & \\
\hline ॥ & $74(52.4)$ & $24(53.3)$ & $50(52.1)$ & \\
\hline III & 45 (31.9) & $15(33.3)$ & $30(31.3)$ & \\
\hline IV & II (7.8) & $3(6.7)$ & $8(8.3)$ & \\
\hline GOLD quadrant & & & & 0.003 \\
\hline$A$ & $9(6.4)$ & $5(11.1)$ & $4(4.2)$ & \\
\hline B & $35(24.8)$ & $7(15.6)$ & $28(29.2)$ & \\
\hline C & $21(14.9)$ & $13(28.9)$ & $8(8.3)$ & \\
\hline $\mathrm{D}$ & 76 (53.9) & $20(44.4)$ & $56(58.3)$ & \\
\hline BMI $\left(\mathrm{kg} / \mathrm{m}^{2}\right)$ & $30.2(25.5,34.7)$ & $30.4(24.5,33.3)$ & $30.2(26.1,35.9)$ & 0.32 \\
\hline Ex-smokers & $116(82.3)$ & $36(80)$ & $80(83.3)$ & 0.64 \\
\hline Pack years & $37.5(13.8,62.5)$ & $35.3(9.8,61.3)$ & $38.4(13.9,63.4)$ & 0.51 \\
\hline SGRQ & $49.7 \pm 16.9$ & $45.7 \pm 18.0$ & $51.6 \pm 16.1$ & 0.052 \\
\hline BODEx & $2(1,5)$ & $2(I, 3)$ & $3(2,5)$ & 0.003 \\
\hline mMRC score $\geq 2$ & $96(68.1)$ & $24(53.3)$ & $72(75.0)$ & 0.01 \\
\hline ICS or ICS/LABA combination use & $128(90.8)$ & $4 I(91.1)$ & $87(90.6)$ & 0.60 \\
\hline ICS dose ${ }^{d}(\mu g / d)$ & $500(250,500)$ & $500(250,500)$ & $500(250,500)$ & 0.74 \\
\hline LAMA use & $110(78.0)$ & $35(77.8)$ & $75(78.1)$ & 0.56 \\
\hline Prior history of asthma & $69(48.9)$ & $23(51.1)$ & $46(47.9)$ & 0.86 \\
\hline
\end{tabular}

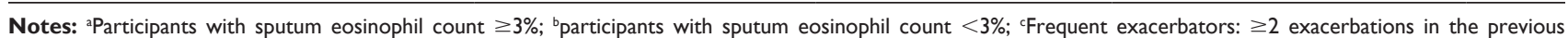
year, and nonfrequent exacerbators: $<2$ exacerbations in the previous year; ${ }^{\mathrm{I}} \mathrm{CS}$ dose calculated as beclomethasone equivalents where I $\mu \mathrm{g}$ of beclomethasone $=\mathrm{I} \mu \mathrm{g}$ budesonide $=0.5 \mu \mathrm{g}$ fluticasone; $* P$-value for the comparison of eosinophilic vs noneosinophilic COPD. Data expressed as mean \pm SD, median (interquartile range) or as number (\%).

Abbreviations: $\mathrm{FEV}_{1}$, forced expiratory volume in I second; FVC, forced vital capacity; CCI, Charlson comorbidity index; GOLD, Global Initiative for Chronic Obstructive Lung Disease; BMI, body mass index; SGRQ, St George Respiratory Questionnaire; BODEx, body mass index, airflow obstruction, dyspnea, severe exacerbation; mMRC, modified Medical Research Council; ICS, inhaled corticosteroids; LABA, long-acting $\beta_{2}$ agonist; LAMA, long-acting muscarinic antagonists; SD, standard deviation.

(Figure 3B). Similarly, percentage sputum eosinophils correlated reasonably well with both blood ELR $(\rho=0.488$; $P<0.0001)$ and blood ENR $(\rho=0.592 ; P<0.0001)$. No significant association was observed between percentage sputum neutrophils and blood neutrophil/lymphocyte ratio ( $\rho=0.0287 ; P=0.7355$ ).

\section{Receiver operating characteristic (ROC) curve analysis}

Absolute blood eosinophil count was predictive of sputum eosinophilia with AUC of 0.76 (95\% confidence interval $[\mathrm{CI}]=0.67-0.84 ; P<0.0001$ ) (Figure 4). Percentage blood eosinophils, blood ELR, and blood ENR were also predictive of

Table 2 Blood cell count parameters and blood cell ratios

\begin{tabular}{lllll}
\hline Blood cell counts/ratios & All participants $(\mathbf{n}=\mathbf{I 4 I})$ & Eosinophilic $^{\mathrm{a}}(\mathbf{n}=\mathbf{4 5})$ & Noneosinophilic $^{\mathrm{b}}(\mathbf{n}=\mathbf{9 6})$ & $\boldsymbol{P}_{\text {-value* }}$ \\
\hline Blood eosinophils $\left(\times 10^{9} / \mathrm{L}\right)$ & $0.20(0.10,0.30)$ & $0.30(0.20,0.40)$ & $0.15(0.10,0.20)$ & $<0.0001$ \\
Blood eosinophils $(\%)$ & $2.70(1.56,3.92)$ & $3.95(2.94,5.08)$ & $2.07(1.35,3.25)$ & $<0.0001$ \\
Blood neutrophils $\left(\times 10^{9} / \mathrm{L}\right)$ & $5.08 \pm 1.67$ & $4.60 \pm 1.53$ & $5.30 \pm 1.70$ & 0.02 \\
White blood cells $\left(\times 10^{9} / \mathrm{L}\right)$ & $7.62 \pm 1.95$ & $7.38 \pm 1.77$ & $7.73 \pm 2.03$ & 0.32 \\
Blood lymphocytes $\left(\times 10^{9} / \mathrm{L}\right)$ & $1.78 \pm 0.65$ & $1.76 \pm 0.58$ & $1.78 \pm 0.68$ & 0.84 \\
Blood NLR & $2.84(2.15,3.85)$ & $2.59(2.07,3.23)$ & $3.00(2.24,4.00)$ & 0.04 \\
Blood ELR & $0.12(0.07,0.18)$ & $0.15(0.13,0.24)$ & $0.09(0.06,0.16)$ & $<0.0001$ \\
Blood ENR & $0.04(0.02,0.06)$ & $0.07(0.04,0.08)$ & $0.03(0.02,0.05)$ & $<0.0001$ \\
\hline
\end{tabular}

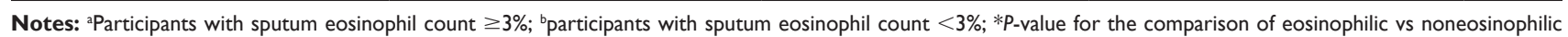
COPD. Data expressed as mean $\pm \mathrm{SD}$, median (interquartile range) or as number (\%).

Abbreviations: NLR, neutrophil/lymphocyte ratio; ELR, eosinophil/lymphocyte ratio; ENR, eosinophil/neutrophil ratio; SD, standard deviation. 

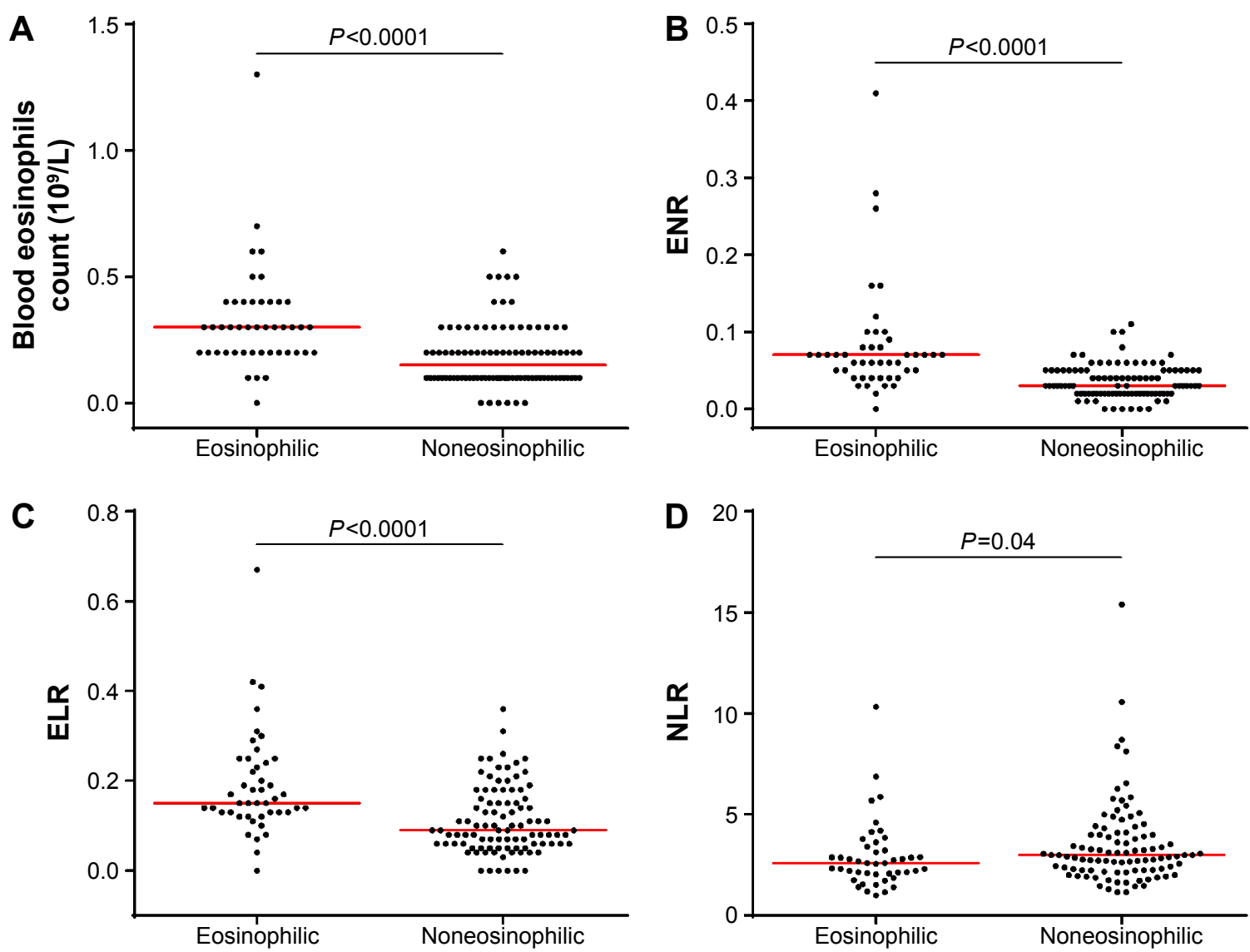

Figure 2 Scatter dot plot comparing.

Notes: (A) Blood eosinophil count, (B) eosinophil/neutrophil ratio, (C) eosinophil/lymphocyte ratio, and (D) neutrophil/lymphocyte ratio between eosinophilic ( $\geq 3 \%$ sputum eosinophils) and noneosinophilic COPD ( $<3 \%$ sputum eosinophils). Graphs represent individual data points and a median as a bar (red).

Abbreviations: ENR, eosinophil/neutrophil ratio; ELR, eosinophil/lymphocyte ratio; NLR, neutrophil/lymphocyte ratio.

sputum eosinophilia with AUCs of $0.80(95 \% \mathrm{CI}=0.73-0.86)$ $0.74(95 \% \mathrm{CI}=0.65-0.83)$, and $0.81(95 \% \mathrm{CI}=0.73-0.89)$, respectively. The sensitivities and specificities of different cutoff points of absolute blood eosinophil counts were evaluated together with their ability to correctly classify patients (Table 3). A summary of sensitivity and specificity for percentage blood eosinophils and blood ENR of different cutoff points is provided in the Supplementary material (Tables S1 and $\underline{\mathrm{S}}$ ). The absolute blood eosinophil count threshold that balanced sensitivity and specificity on the ROC curve was found to be $\geq 0.3 \times 10^{9} / \mathrm{L}(300 / \mu \mathrm{L})$, with a sensitivity of $60 \%$, specificity of $76 \%$, and a positive likelihood ratio of 2.5 .
A

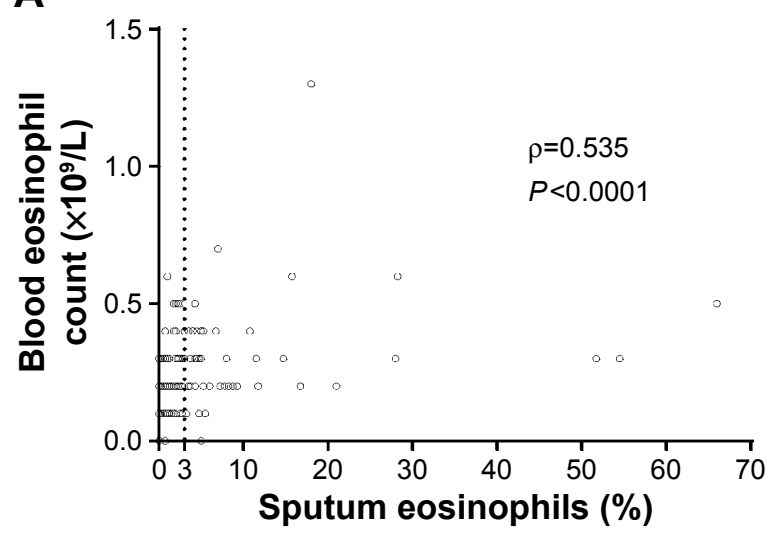

B

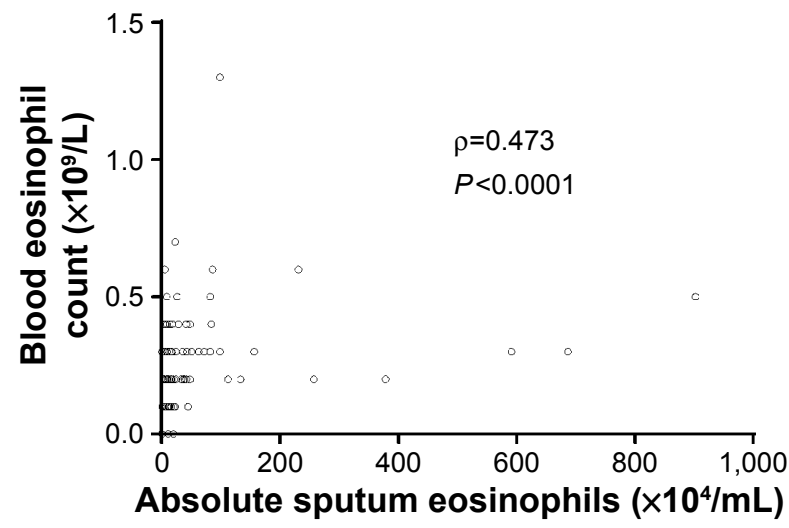

Figure 3 Scatter plots for correlations between sputum and blood eosinophil counts.

Notes: (A) Correlation between percentage sputum eosinophils and absolute blood eosinophil count $\left(\times 10^{9} / \mathrm{L}\right)$. Vertical dotted line represents upper limit of normal for percentage sputum eosinophils. (B) Correlation between absolute sputum eosinophil counts $\left(\times 10^{4} / \mathrm{L}\right)$ and absolute blood eosinophil count $\left(\times 10^{9} / \mathrm{L}\right)$. 


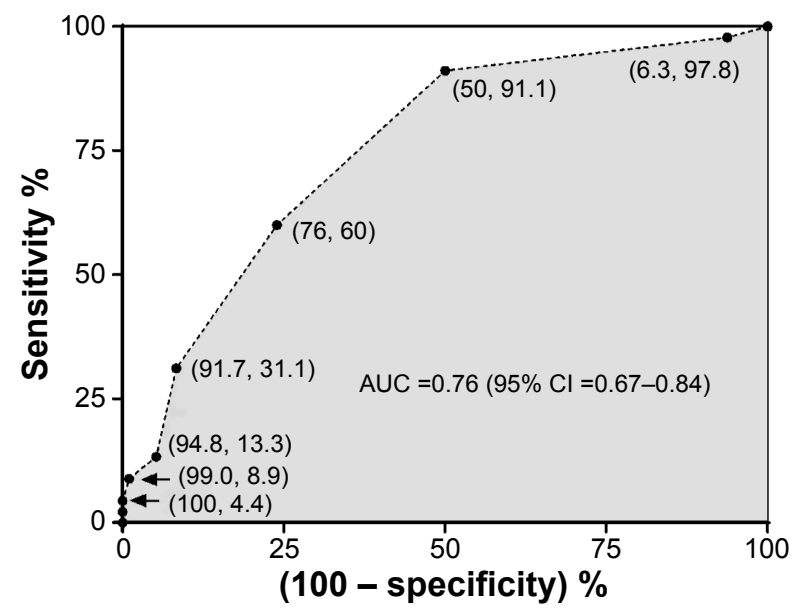

Figure 4 Receiver operating characteristic (ROC) curve for absolute blood eosinophil count to predict sputum eosinophilia ( $\geq 3 \%$ ).

Note: Points on the ROC curve are presented as (specificity \%, sensitivity \%).

At this cutoff point, blood eosinophil counts enabled the correct identification of the presence or absence of sputum eosinophilia in 71 cases out of 100 . A higher cutoff point of $\geq 0.4 \times 10^{9} / \mathrm{L}$ $(400 / \mu \mathrm{L})$ gave a greater specificity $(91.7 \%)$, a higher positive likelihood ratio (3.7), and an essentially similar classifying ability. In contrast, a higher degree of sensitivity (91.1\%) was achieved at a peripheral blood eosinophil cutoff point of $0.2 \times 10^{9} / \mathrm{L}(200 / \mu \mathrm{L})$.

Based on the peripheral blood eosinophil threshold of $\geq 0.3 \times 10^{9} / \mathrm{L}, 76 \%$ of the noneosinophilic participants (73 out of 96) would be correctly characterized as not having sputum eosinophilia (true negatives) while the remaining $24 \%$ as false positives. On the other hand, $60 \%$ of the eosinophilic participants ( 27 out of 45 ) would be accurately identified as having sputum eosinophilia (true positives) while the remaining $40 \%$ as false negatives. Two-by-two contingency tables for the blood eosinophil cutoff points of $\geq 0.2 \times 10^{9} / \mathrm{L}$ and $\geq 0.4 \times 10^{9} / \mathrm{L}$ are provided in the Supplementary material (Tables $\mathrm{S} 3$ and $\underline{\mathrm{S}} 4$ ).
Clinical characteristics of participants classified by blood eosinophil counts

Blood eosinophilia (blood eosinophil count $\geq 0.4 \times 10^{9} / \mathrm{L}$ ) was present in $22(15.6 \%)$ participants (Table 4). Patients with blood eosinophilia had a higher postbronchodilator $\mathrm{FEV}_{1} \%$ predicted and a lower BODEx score compared to those without $\left(<0.4 \times 10^{9} / \mathrm{L}\right)$. All other clinical parameters were similar between the two groups. There were no differences in blood eosinophil counts between males and females $\left(0.2[0.1,0.3] \times 10^{9} / \mathrm{L}\right.$ and $\left.0.2[0.1,0.3] \times 10^{9} / \mathrm{L} ; P=0.9087\right)$, ex-smokers and never smokers $\left(0.2[0.1,0.3] \times 10^{9} / \mathrm{L}\right.$ and $\left.0.2[0.1,0.3] \times 10^{9} / \mathrm{L} ; P=0.8848\right)$, frequent and nonfrequent exacerbators $\left(0.2[0.1,0.3] \times 10^{9} / \mathrm{L}\right.$ and $0.2[0.1,0.3] \times 10^{9} / \mathrm{L}$; $P=0.96$ ), or between patients taking ICS(/LABA) and those who did not $\left(0.2[0.1,0.3] \times 10^{9} / \mathrm{L}\right.$ and $0.1[0.1,0.2] \times 10^{9} / \mathrm{L}$; $P=0.221)$.

\section{Stability study}

This study also assessed the stability of peripheral blood eosinophil counts in those participants who had repeated measurements of blood cell counts obtained from blood samples collected at two visits spaced a median of $28(22.5,35.5)$ days apart $(n=46)$. There was a good agreement between the two measurements, with an intraclass correlation coefficient of $0.8(95 \% \mathrm{CI}=0.66-0.88 ; P<0.0001)$. The bias of measurement was negligible $\left(0.002 \pm 0.13\left[\times 10^{9} / \mathrm{L}\right]\right)$, with equal scatter around the bias line, indicating no systematic measurement bias (Figure 5).

\section{Discussion}

This study, which assessed the ability of peripheral blood eosinophils for detecting sputum eosinophilia in stable COPD, had three main findings. First, peripheral blood eosinophil count was shown to distinguish patients with sputum eosinophilia from those without, thereby indicating its potential use as a diagnostic biomarker for eosinophilic COPD. Second,

Table 3 Summary of sensitivity and specificity for different blood eosinophil cutoff points for detecting sputum eosinophilia

\begin{tabular}{|c|c|c|c|c|c|}
\hline $\begin{array}{l}\text { Blood eosinophil cutoff } \\
\text { points }\left(\times 10^{9} / L\right)\end{array}$ & Sensitivity (\%) & Specificity (\%) & $\begin{array}{l}\text { Correctly } \\
\text { classified (\%) }\end{array}$ & LR (+) & LR (-) \\
\hline$\geq 0.1$ & 97.8 & 6.3 & 35.5 & 1.04 & 0.36 \\
\hline$\geq 0.2$ & 91.1 & 50.0 & 63.1 & 1.82 & 0.18 \\
\hline$\geq 0.3$ & 60.0 & 76.0 & 70.9 & 2.50 & 0.53 \\
\hline$\geq 0.4$ & 31.1 & 91.7 & 72.3 & 3.73 & 0.75 \\
\hline$\geq 0.5$ & 13.3 & 94.8 & 68.8 & 2.56 & 0.91 \\
\hline$\geq 0.6$ & 8.9 & 99.0 & 70.2 & 8.53 & 0.92 \\
\hline$\geq 0.7$ & 4.4 & 100.0 & 69.5 & a & 0.96 \\
\hline
\end{tabular}

Note: ${ }^{2}$ The LR (+) at this cutoff point is incalculable (with 0 as a denominator).

Abbreviations: LR (+), positive likelihood ratio; LR (-), negative likelihood ratio. 
Table 4 Demographics and clinical characteristics of participants with and without blood eosinophilia

\begin{tabular}{|c|c|c|c|}
\hline Variable & $\begin{array}{l}\text { Participants with blood } \\
\left.\text { eosinophilia ( } \geq 0.4 \times 10^{9} / \mathrm{L}\right)\end{array}$ & $\begin{array}{l}\text { Participants without blood } \\
\left.\text { eosinophilia ( }<0.4 \times 10^{9} / \mathrm{L}\right)\end{array}$ & $P$-value* \\
\hline Number & $22(15.6)$ & $119(84.4)$ & NA \\
\hline Age (years) & $70.1 \pm 7.5$ & $69.7 \pm 7.8$ & 0.84 \\
\hline Sex (male) & $13(59.1)$ & $76(63.9)$ & 0.81 \\
\hline Postbronchodilator FEV (\% predicted) & $65.1 \pm 19.3$ & $56.1 \pm 17.4$ & 0.03 \\
\hline Postbronchodilator FEV (L) & $1.56(1.2,1.88)$ & $1.48(1.12,1.79)$ & 0.31 \\
\hline Postbronchodilator $\mathrm{FEV}_{1} / \mathrm{FVC}$ ratio (\%) & $58.82 \pm 14.83$ & $54.64 \pm 14.79$ & 0.23 \\
\hline Reversibility (L) & $0.025(0,0.12)$ & $0.045(0.01,0.1)$ & 0.51 \\
\hline Reversibility (\%) & $2.5(0,12.03)$ & $4.55(0.95,9.66)$ & 0.51 \\
\hline $\mathrm{CCl}$ score & $4(3,5)$ & $4(3,4)$ & 0.14 \\
\hline Frequent vs nonfrequent exacerbators ${ }^{\mathrm{a}}$ & 13 vs $9(59.1,40.9)$ & 6 I vs $58(51.2,48.8)$ & 0.64 \\
\hline Number of severe exacerbation & $0(0,1)$ & $0(0,1)$ & 0.60 \\
\hline GOLD grade & & & 0.23 \\
\hline I & $4(18.2)$ & $7(5.9)$ & \\
\hline II & $12(54.5)$ & $62(52.1)$ & \\
\hline III & $5(22.7)$ & $40(33.6)$ & \\
\hline IV & I (4.5) & $10(8.4)$ & \\
\hline GOLD quadrant & & & 0.16 \\
\hline$A$ & $0(0)$ & $9(7.6)$ & \\
\hline B & $3(13.6)$ & $32(26.9)$ & \\
\hline $\mathrm{C}$ & $6(27.3)$ & $15(12.6)$ & \\
\hline $\mathrm{D}$ & $13(59.1)$ & $63(52.9)$ & \\
\hline BMI $\left(\mathrm{kg} / \mathrm{m}^{2}\right)$ & $31.1(26.7,34.5)$ & $30.2(25.5,34.9)$ & 0.91 \\
\hline Ex-smokers & $19(86.4)$ & $97(81.5)$ & 0.77 \\
\hline Pack years & $22.3(9,42)$ & $38.8(15,65)$ & 0.06 \\
\hline SGRQ & $44.2(32.8,66.6)$ & $50.2(38,61.1)$ & 0.89 \\
\hline BODEX & $2(I, 3)$ & $3(I, 5)$ & 0.047 \\
\hline $\mathrm{mMRC}$ score $\geq 2$ & $12(54.5)$ & $84(59.6)$ & 0.14 \\
\hline ICS or ICS/LABA combination use & $22(100)$ & $106(89.1)$ & 0.22 \\
\hline ICS dose ${ }^{b}(\mu g / d)$ & $500(400,500)$ & $500(250,500)$ & 0.16 \\
\hline LAMA use & $16(72.7)$ & $94(79)$ & 0.52 \\
\hline Prior history of asthma & $12(55)$ & $57(48)$ & 0.65 \\
\hline
\end{tabular}

Notes: aFrequent exacerbators: $\geq 2$ exacerbations in the previous year, and nonfrequent exacerbators: $<2$ exacerbations in the previous year; bاCS dose calculated as beclomethasone equivalents where I $\mu \mathrm{g}$ of beclomethasone $=\mathrm{I} \mu \mathrm{g}$ budesonide $=0.5 \mu \mathrm{g}$ fluticasone. Data expressed as mean $\pm \mathrm{standard}$ deviation, median (interquartile range) or as number (\%); *P-value for the comparison of participants with and without blood eosinophilia.

Abbreviations: FEV , forced expiratory volume in I second; FVC, forced vital capacity; CCl, Charlson Comorbidity Index; GOLD, Global Initiative for Chronic Obstructive Lung Disease; BMI, body mass index; SGRQ, St George Respiratory Questionnaire; BODEx, body mass index, airflow obstruction, dyspnea, severe exacerbation; mMRC, modified Medical Research Council; ICS, inhaled corticosteroids; LABA, long-acting $\beta_{2}$ agonist; LAMA, long-acting muscarinic antagonists; NA, not applicable.

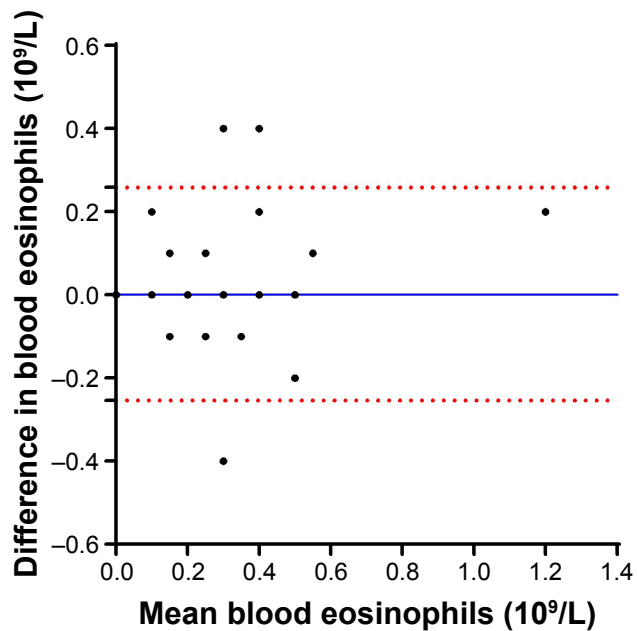

Figure 5 Bland-Altman plot showing the difference between the absolute blood eosinophil counts of two measurements against the mean of the absolute blood eosinophil counts of the two measurements.

Notes: The blue line represents the mean of differences (bias line). Horizontal dotted lines represent the $95 \%$ limits of agreement (mean difference $\pm 1.96 \mathrm{SD}$ ). Abbreviation: SD, standard deviation. we have shown that blood eosinophil counts and their ratios (ELR and ENR) are elevated in eosinophilic COPD and correlate reasonably well with sputum eosinophilia. Third, blood eosinophil counts between two measurements over a median period of 28 days were found to be stable.

The diagnostic performance of peripheral blood eosinophils in identifying eosinophilic airway inflammation in mild, moderate, and severe asthma has been previously investigated. ${ }^{13-18,34}$ Studies examining the potential utility of blood eosinophils in COPD, particularly during stable conditions, are however few. ${ }^{3,19-21}$ One study in COPD has shown that peripheral percentage blood eosinophil count $(>2 \%)$ can serve as a sensitive biomarker to determine sputum eosinophilia ( $>3 \%$ ) during exacerbations (AUC 0.85 $[95 \% \mathrm{CI}=0.78-0.93]$, sensitivity $=90 \%$, specificity $=60 \%) .{ }^{4}$ This AUC result is similar to our present AUC result in stable COPD. 
As noted by Korevaar et al, ${ }^{14}$ optimal cutoff points for diagnostic biomarkers of airway eosinophilia selected by balancing sensitivity and specificity on a ROC curve may not be clinically applicable, given that their sensitivity and/or specificity is often suboptimal compared to that of reference standard tests, such as bronchoalveolar lavage and sputum induction. From the clinical point of view, the choice of a cutoff point is also partly determined by the clinical question. In view of this, we have evaluated the sensitivity and specificity of blood eosinophil counts at different cutoff points (Table 3 ). According to our data, in patients with stable COPD, peripheral blood eosinophil counts can help correctly identify the presence or absence of sputum eosinophilia in 71 cases out of 100 at cutoff points of $\geq 0.3 \times 10^{9} / \mathrm{L}$ or $\geq 0.4 \times 10^{9} / \mathrm{L}$. Nevertheless, the higher cutoff point had a higher positive predictive value (PPV) (number of true positives/[number of true positive + number of false positives]) and a much better specificity to rule in sputum eosinophilia. This implies that patients with blood eosinophil counts above the threshold of $0.4 \times 10^{9} / \mathrm{L}$ would most likely have COPD with eosinophilic airway inflammation. Akin to the suggestion of Fowler et al, ${ }^{17}$ such patients may not necessarily need to undergo induction of sputum for the assessment of airway eosinophilia. Patients with blood eosinophil count of $<0.4 \times 10^{9} / \mathrm{L}$ may however need further assessment because the proportion of false negatives at the cutoff point of $0.4 \times 10^{9} / \mathrm{L}$ is high $(\sim 69 \%)$. As a matter of interest, a similar blood eosinophil cutoff point of $\geq 0.45 \times 10^{9} / \mathrm{L}$ has been reported to help correctly identify sputum eosinophilia in patients with severe asthma with a specificity of $97 \%$, sensitivity of $49.3 \%$, and PPV of 89.2. ${ }^{17}$ Another recent study has also reported a blood eosinophil cutoff point of $\geq 0.41 \times 10^{9} / \mathrm{L}$ (specificity of $95 \%$, sensitivity of $36 \%$, and PPV of 79 ) for detecting sputum eosinophilia in a population of asthmatic patients of different phenotypes. ${ }^{15}$

For a clinician who wants to rule out sputum eosinophilia, on the other hand, a peripheral blood eosinophil cutoff point with a high sensitivity would be of interest. Based on our results, patients are unlikely to have sputum eosinophilia if their blood eosinophil count is below $0.2 \times 10^{9} / \mathrm{L}$ because the sensitivity at the cutoff point of $0.2 \times 10^{9} / \mathrm{L}$ is $91.1 \%$.

It is interesting to note that in our study, there was discordance between sputum and blood eosinophils in approximately $40 \%$ of the patients with eosinophilic COPD at a peripheral blood eosinophil cutoff point of $0.3 \times 10^{9} / \mathrm{L}$. This observation is similar to that of a recent study in patients with uncontrolled asthma where one-third of the participants exhibited discordance between blood and sputum eosinophils. ${ }^{35}$ In order to gain a better insight into our true-positive
(27 patients) and false-negative (18 patients) cohorts, their demographic features, clinical characteristics, and sputum cell counts were compared. The results nevertheless revealed no significant difference between the two groups (Table S5). However, it should be highlighted that this analysis may have been underpowered due to small sample size.

Plausible causes for the discordance between sputum and blood eosinophils may include the imbalance between the production and subsequent clearance of eosinophils by airway macrophages ${ }^{36}$ and variations in the process of recruitment of eosinophils into the airways. ${ }^{35}$ The fact that only a proportion of the eosinophilic COPD patients had blood eosinophilia may suggest the involvement of more than one distinct biological mechanisms underlying eosinophilic airway inflammation within this COPD phenotype ${ }^{37}$ As in the case of asthma, Th2 cytokines could be responsible for inflammation in eosinophilic COPD. ${ }^{37}$ Nevertheless, eosinophilic airway inflammation in the absence of elevated levels of Th2 has also been reported in some COPD patients. ${ }^{38}$ One potential mechanism for the non-Th2 eosinophilic inflammation in COPD could be the epithelial-innate lymphoid cell type 2 (ILC2) pathway, which has been suggested to play a similar role in severe nonallergic asthma. ${ }^{39}$ Obviously, further investigation is warranted to understand the mechanism underlying the different endotypes of the eosinophilic COPD phenotype.

Our data demonstrated that blood eosinophils and their ratios (ELR and ENR) were elevated in patients with sputum eosinophilia compared with those without. Absolute blood eosinophil counts correlated reasonably well with both the absolute sputum counts and percentage sputum eosinophils, which is in agreement with the findings of Wagener et al, ${ }^{18}$ Zhang et al, ${ }^{16}$ and Fowler et a ${ }^{17}$ in asthma, but not with those of Hastie et $\mathrm{al}^{34}$ and Amorim et $\mathrm{al} .^{40} \mathrm{In}$ accordance with a previous finding in asthma, ${ }^{16}$ in our study, both blood ENR and ELR also correlated with percentage sputum eosinophils and were predictive of eosinophilic COPD with AUCs of 0.81 and 0.74 , respectively. Recently, Khatry et $\mathrm{al}^{41}$ have suggested that ratios of blood cell types, such as ELR and ENR, may minimize variations associated with measurement, sample processing, and therapies and yield a more accurate diagnostic performance over actual blood cell counts. This certainly will be a topical issue for future studies in COPD.

According to Price et $\mathrm{al},{ }^{42}$ blood eosinophilia (defined as $\geq 0.5 \times 10^{9} / \mathrm{L}$ ) occurs in $10 \%$ of stable COPD patients and is associated with higher rate of exacerbations, particularly in nonsmokers receiving maintenance therapy. Elevated levels of blood eosinophils in COPD patients have also been associated 
with increased risk of mortality from exacerbations. ${ }^{43} \mathrm{Nev}$ ertheless, our analysis indicated no significant difference in blood eosinophil counts between frequent and nonfrequent exacerbators. Similarly, there was no difference in the number of severe exacerbations in the past 12 months between patients with blood eosinophilia $\left(\geq 0.4 \times 10^{9} / \mathrm{L}\right)$ and those without $\left(<0.4 \times 10^{9} / \mathrm{L}\right)$. It is worth noting here that up to $90 \%$ of our study population was using either ICS/LABA alone or in combination with LAMA, which are known to reduce the risk of exacerbation. All things considered, the aforementioned discrepancies may possibly be due to differences in the characteristics of study populations.

In our study, the stability of peripheral blood eosinophil counts between two measurements over a median period of 28 days was found to be acceptable. This implies that a single measurement of blood eosinophil count may provide indicative information about eosinophilic airway inflammation status in stable COPD. The stability of peripheral blood eosinophil counts in repeated measurements in COPD population has been reported in other studies as well. ${ }^{20,21}$

\section{Conclusion}

In this study, we found a predictive relationship between blood and sputum eosinophils in stable COPD. Peripheral blood eosinophils were stable between two measurements, suggesting that a single blood eosinophil count may potentially serve as a reliable marker for eosinophilic COPD. A potential limitation of this study may be the fact that we did not have bronchoalveolar lavage or endobronchial biopsy samples for the assessment of airway eosinophilia.

The clinical relevance of our work lies in the fact that a simple blood test may allow the clinician to positively diagnose eosinophilic COPD. In relation to this, the question of when to use ICS in COPD is of high clinical importance. Biomarkers that allow for the identification of patients who are most likely to respond to ICS, and consequently minimize harm arising from inappropriate treatment, have the potential to significantly progress COPD management. In this regard, it will be interesting and a worthwhile endeavor to examine ICS response at different blood eosinophil thresholds in future prospective studies.

\section{Acknowledgments}

This study was supported by National Health and Medical Research Council (NHMRC), Australia, Grant ID: 1045230 (PGG, VMM, JLS, PABW); Ramaciotti Foundation (VMM); Lung Foundation of Australia (VMM); National Health and Medical Research Council (NHMRC), Australia, Grant
ID: 455508 (2007-2010); and Priority Research Centre for Asthma and Respiratory Diseases PhD Scholarship and Emlyn and Jennie Thomas Postgraduate Medical Research Scholarship through the Hunter Medical Research Institute (NAN). The authors wish to acknowledge Kelly Steel, Amber Smith, Hayley Lunn, Penny Baines, Gabrielle LeBrocq, Brooke Emmett, Clare Powell, and Hayley Candler for their role in data collection; Kellie Fakes, Bridgette Donati, and Michelle Gleeson for their role in sample processing; and Heather Powell for statistical advice.

\section{Disclosure}

VMM has received grants from Ramaciotti Foundation, NHMRC, Lung Foundation of Australia during the conduct of the study; personal fees and honoraria for education and advisory boards from Menarini and personal fees from GSK, Novartis, and Astra Zeneca outside this work. KJB reports research fellowship from The Thoracic Society of Australia and New Zealand/National Asthma council. PGG is supported by a NHMRC practitioner fellowship and has received speakers fees from GlaxoSmithKline, Boehringer Ingelheim, AstraZeneca, and Novartis outside this work. PWJ reports being employed as a global medical expert by GSK. The authors report no other conflicts of interest in this work.

\section{References}

1. Saha S, Brightling CE. Eosinophilic airway inflammation in COPD. Int J Chron Obstruct Pulmon Dis. 2006;1(1):39-47.

2. Brightling CE. Clinical applications of induced sputum. Chest. 2006; 129(5):1344-1348.

3. Singh D, Kolsum U, Brightling CE, Locantore N, Agusti A, Tal-Singer R; for ECLIPSE investigators. Eosinophilic inflammation in COPD: prevalence and clinical characteristics. Eur Respir J. 2014;44(6): $1697-1700$.

4. Bafadhel M, McKenna S, Terry S, et al. Acute exacerbations of chronic obstructive pulmonary disease: identification of biologic clusters and their biomarkers. Am J Respir Crit Care Med. 2011;184(6): 662-671.

5. McDonald VM, Higgins I, Wood LG, Gibson PG. Multidimensional assessment and tailored interventions for COPD: respiratory utopia or common sense? Thorax. 2013;68(7):691-694.

6. Leigh R, Pizzichini MM, Morris MM, Maltais F, Hargreave FE, Pizzichini E. Stable COPD: predicting benefit from high-dose inhaled corticosteroid treatment. Eur Respir J. 2006;27(5):964-971.

7. Brightling CE, Monteiro W, Ward R, et al. Sputum eosinophilia and shortterm response to prednisolone in chronic obstructive pulmonary disease: a randomised controlled trial. Lancet. 2000;356(9240):1480-1485.

8. Brightling CE, McKenna S, Hargadon B, et al. Sputum eosinophilia and the short term response to inhaled mometasone in chronic obstructive pulmonary disease. Thorax. 2005;60(3):193-198.

9. Pizzichini E, Pizzichini MM, Gibson P, et al. Sputum eosinophilia predicts benefit from prednisone in smokers with chronic obstructive bronchitis. Am J Respir Crit Care Med. 1998;158(5 Pt 1): 1511-1517.

10. Pavord ID, Bafadhel M. Exhaled nitric oxide and blood eosinophilia: independent markers of preventable risk. J Allergy Clin Immunol. 2013; 132(4):828-829. 
11. Baines KJ, Pavord ID, Gibson PG. The role of biomarkers in the management of airways disease. Int J Tuberc Lung Dis. 2014;18(11): 1264-1268.

12. Pavord ID, Gibson PG. Inflammometry: the current state of play. Thorax. 2012;67(3):191-192.

13. Yap E, Chua WM, Jayaram L, Zeng I, Vandal AC, Garrett J. Can we predict sputum eosinophilia from clinical assessment in patients referred to an adult asthma clinic? Intern Med J. 2013;43(1):46-52.

14. Korevaar DA, Westerhof GA, Wang J, et al. Diagnostic accuracy of minimally invasive markers for detection of airway eosinophilia in asthma: a systematic review and meta-analysis. Lancet Respir Med. 2015;3(4): 290-300.

15. Westerhof GA, Korevaar DA, Amelink M, et al. Biomarkers to identify sputum eosinophilia in different adult asthma phenotypes. Eur Respir J. 2015;46(3):688-696.

16. Zhang XY, Simpson JL, Powell H, et al. Full blood count parameters for the detection of asthma inflammatory phenotypes. Clin Exp Allergy. 2014;44(9):1137-1145.

17. Fowler SJ, Tavernier G, Niven R. High blood eosinophil counts predict sputum eosinophilia in patients with severe asthma. J Allergy Clin Immunol. 2015;135(3):822-824.e822.

18. Wagener AH, de Nijs SB, Lutter R, et al. External validation of blood eosinophils, FE (NO) and serum periostin as surrogates for sputum eosinophils in asthma. Thorax. 2015;70(2):115-120.

19. Eltboli O, Mistry V, Barker B, Brightling CE. Relationship between blood and bronchial submucosal eosinophilia and reticular basement membrane thickening in chronic obstructive pulmonary disease. Respirology. 2015;20(4):667-670.

20. Bafadhel M, McKenna S, Terry S, et al. Blood eosinophils to direct corticosteroid treatment of exacerbations of chronic obstructive pulmonary disease: a randomized placebo-controlled trial. Am J Respir Crit Care Med. 2012;186(1):48-55.

21. Pascoe S, Locantore N, Dransfield MT, Barnes NC, Pavord ID. Blood eosinophil counts, exacerbations, and response to the addition of inhaled fluticasone furoate to vilanterol in patients with chronic obstructive pulmonary disease: a secondary analysis of data from two parallel randomised controlled trials. Lancet Respir Med. 2015;3(6):435-442.

22. Siddiqui SH, Guasconi A, Vestbo J, et al. Blood eosinophils: a biomarker of response to extrafine beclomethasone/formoterol in chronic obstructive pulmonary disease. Am J Respir Crit Care Med. 2015;192(4): 523-525.

23. Simpson JL, Powell H, Baines KJ, et al. The effect of azithromycin in adults with stable neutrophilic COPD: a double blind randomised, placebo controlled trial. PLoS One. 2014;9(8):e105609.

24. McDonald V, Gibson PG, Scott H, et al. Should we treat obesity in COPD? The effects of diet and exercise training. Respirology. 2016; doi:10.1111/resp.12746.

25. Bestall JC, Paul EA, Garrod R, Garnham R, Jones PW, Wedzicha JA. Usefulness of the Medical Research Council (MRC) dyspnoea scale as a measure of disability in patients with chronic obstructive pulmonary disease. Thorax. 1999;54(7):581-586.

26. Charlson ME, Pompei P, Ales KL, MacKenzie CR. A new method of classifying prognostic comorbidity in longitudinal studies: development and validation. J Chronic Dis. 1987;40(5):373-383.
27. Jones PW, Quirk FH, Baveystock CM, Littlejohns P. A self-complete measure of health status for chronic airflow limitation. The St George's respiratory questionnaire. Am Rev Respir Dis. 1992;145(6):1321-1327.

28. Soler-Cataluna JJ, Martinez-Garcia MA, Sanchez LS, Tordera MP, Sanchez PR. Severe exacerbations and BODE index: two independent risk factors for death in male COPD patients. Respir Med. 2009;103(5): 692-699.

29. Standardization of Spirometry, 1994 Update. American Thoracic Society. Am J Respir Crit Care Med. 1995;152(3):1107-1136.

30. Hankinson JL, Odencrantz JR, Fedan KB. Spirometric reference values from a sample of the general US population. Am J Respir Crit Care Med. 1999;159(1):179-187.

31. Gibson PG, Wlodarczyk JW, Hensley MJ, et al. Epidemiological association of airway inflammation with asthma symptoms and airway hyperresponsiveness in childhood. Am J Respir Crit Care Med. 1998;158(1): 36-41.

32. Fahy JV, Boushey HA, Lazarus SC, et al. Safety and reproducibility of sputum induction in asthmatic subjects in a multicenter study. Am J Respir Crit Care Med. 2001;163(6):1470-1475.

33. Pavord ID, Brightling CE, Woltmann G, Wardlaw AJ. Non-eosinophilic corticosteroid unresponsive asthma. Lancet. 1999;353(9171):2213-2214.

34. Hastie AT, Moore WC, Li H, et al. Biomarker surrogates do not accurately predict sputum eosinophil and neutrophil percentages in asthmatic subjects. J Allergy Clin Immunol. 2013;132(1):72-80.

35. Schleich FN, Chevremont A, Paulus V, et al. Importance of concomitant local and systemic eosinophilia in uncontrolled asthma. Eur Respir J. 2014;44(1):97-108.

36. Kulkarni NS, Hollins F, Sutcliffe A, et al. Eosinophil protein in airway macrophages: a novel biomarker of eosinophilic inflammation in patients with asthma. J Allergy Clin Immunol. 2010;126(1):61-69.e63.

37. Woodruff PG, Agusti A, Roche N, Singh D, Martinez FJ. Current concepts in targeting chronic obstructive pulmonary disease pharmacotherapy: making progress towards personalised management. Lancet. 2015;385(9979):1789-1798.

38. Ghebre MA, Bafadhel M, Desai D, et al. Biological clustering supports both "Dutch" and "British" hypotheses of asthma and chronic obstructive pulmonary disease. J Allergy Clin Immunol. 2015;135(1):63-72.e10.

39. Brusselle G, Bracke K. Targeting immune pathways for therapy in asthma and chronic obstructive pulmonary disease. Ann Am Thorac Soc. 2014;11(Suppl 5):S322-S328.

40. Amorim MM, Fernandes PB, Caetano LB, Dracoulakis S, Santoro IL, Fernandes AL. Nasal lavage is better than blood count in predicting sputum eosinophilia. Clin Exp Allergy. 2015;45(5):1006-1008.

41. Khatry DB, Gossage DL, Geba GP, et al. Discriminating sputumeosinophilic asthma: accuracy of cutoffs in blood eosinophil measurements versus a composite index, ELEN. J Allergy Clin Immunol. 2015;136(3):812-814.e812.

42. Price D, Rigazio A, Postma D, et al. Blood eosinophilia and the number of exacerbations in COPD patients [abstract]. ERJ. 2014;44(Suppl 58): 4416.

43. Hospers JJ, Schouten JP, Weiss ST, Rijcken B, Postma DS. Asthma attacks with eosinophilia predict mortality from chronic obstructive pulmonary disease in a general population sample. Am J Respir Crit Care Med. 1999;160(6):1869-1874.
International Journal of COPD

\section{Publish your work in this journal}

The International Journal of COPD is an international, peer-reviewed journal of therapeutics and pharmacology focusing on concise rapid reporting of clinical studies and reviews in COPD. Special focus is given to the pathophysiological processes underlying the disease, intervention programs, patient focused education, and self management protocols.
Dovepress

This journal is indexed on PubMed Central, MedLine and CAS. The manuscript management system is completely online and includes a very quick and fair peer-review system, which is all easy to use. Visit http://www.dovepress.com/testimonials.php to read real quotes from published authors. 